\title{
Quintessence cosmology and the cosmic coincidence
}

\author{
S. A. Bludman* \\ Deutsches Elektronen-Synchrotron DESY, Hamburg, Germany \\ and University of Pennsylvania, Philadelphia, Pennsylvania \\ M. $\operatorname{Roos}^{\dagger}$ \\ Division of High Energy Physics, University of Helsinki, Helsinki, Finland
}

(Received 17 October 2001; published 11 January 2002)

\begin{abstract}
Within present constraints on the observed smooth energy and its equation of state parameter $w_{Q}=P / \rho_{Q}$, it is important to find out whether the smooth energy is static (cosmological constant) or dynamic (quintessence). The most dynamical quintessence fields observationally allowed are now still fast rolling and no longer satisfy the tracker approximation if the equation of state parameter varies moderately with cosmic scale $a=1 / 1+z$. We are optimistic about distinguishing between a cosmological constant and appreciably dynamic quintessence by measuring average values for the effective equation of state parameter $w_{Q}(a)$. However, reconstructing the quintessence potential from observations of any scale dependence $w_{Q}(a)$ appears problematic in the near future. For our flat universe, at present dominated by smooth energy in the form of either a cosmological constant (LCDM) or quintessence (QCDM), we calculate the asymptotic collapsed mass fraction to be maximal at the observed smooth energy-matter ratio $\mathcal{R}_{0} \sim 2$. Identifying this collapsed fraction as a conditional probability for habitable galaxies, we infer that the prior distribution is flat in $\mathcal{R}_{0}$ or $\Omega_{m 0}$. Interpreting this prior as a distribution over theories, rather than as a distribution over unobservable subuniverses, leads us to heuristic predictions about the class of future quasistatic quintessence potentials.
\end{abstract}

DOI: $10.1103 /$ PhysRevD.65.043503

PACS number(s): $98.80 . \mathrm{Cq}$

\section{A LOW-DENSITY ACCELERATING FLAT UNIVERSE}

Observations of high-redshift type Ia supernovae (SNIa) [1] show that the expanding Universe is today accelerating, and was most likely preceded by a period of deceleration [2]. Recent observations of the cosmic microwave background have shown that the density of clustered matter is low, $\Omega_{m 0} \sim 1 / 3$, and that the Universe is flat to high precision, $\Omega_{0}=0.99 \pm 0.03$ [3]. Together with the SNIa data, this implies for the remaining smooth energy $\Omega_{Q 0} \sim 2 / 3$. The flat cosmology is therefore determined by one parameter, the present ratio of smooth to clustered energy, $\mathcal{R}_{0} \equiv \Omega_{Q 0} / \Omega_{m 0}$ $=2.2_{-0.5}^{+0.7} \sim 2$ [4], and by the equation of state $P=P\left(\rho_{Q}, a\right)$, which changes with cosmological scale $a$.

The conventionally defined deceleration parameter $-a \ddot{a} / \dot{a}^{2}$ is thus, at present,

$$
q_{0}=\left(1+3 w_{Q 0} \Omega_{Q 0}\right) / 2<0 \quad \text { or } \quad w_{Q 0}<-1 / 3 \Omega_{Q 0} .
$$

For $\Omega_{Q 0}=2 / 3$, this requires $-1 \leqslant w_{Q 0}<-1 / 2$. (SNIa data have recently established a slightly stronger limit $w_{Q}$ $<-0.55$ at $95 \%$ confidence [5].) Within these limits, we will be concerned to distinguish between static smooth energy $w_{Q}=-1$ and the other limiting case $w_{Q 0}=-0.5$, the maximum value for which the present universe will be accelerating [6].

The observations thus require smooth energy $\rho_{Q}$ with negative pressure $P=w_{Q} \rho_{Q}$. If this is quantum vacuum energy, its small present value would require fine-tuning to 54

\footnotetext{
*Electronic address: bludman@mail.desy.de

†Electronic address: Matts.Roos@helsinki.fi
}

decimals. More plausibly, we assume the vacuum energy to vanish exactly, due to some unknown symmetry principle, and invent some dynamical mechanism for antigravitating smooth energy, slowly varying on a Hubble scale. This explains how a huge cosmological "constant" could become small now, but does not fix the one cosmological parameter $\mathcal{R}_{0} \sim 2$. Unless totally accidental, the "cosmic coincidence" of our presence just when the dark energy is dominating the background energy must derive either from a still-to-be discovered quantum cosmology or from some selection effect connected with our existence (anthropic principle).

By quintessence $[7,8]$ we mean a rolling scalar field $\phi$ which, for a large class of initial conditions, converges towards a tracker field obeying an effective "equation of state" (EOS) $w_{Q}(a)=P / \rho_{Q}$ with $P \equiv P_{Q}\left(\rho_{Q}, a\right)$, which tracks the background field "equation of state" $w_{B}(a)$. The background EOS $w_{B}(a)=P_{\text {rad }} /\left(\rho_{\text {rad }}+\rho_{\text {mat }}\right)$ reduces gradually from $1 / 3$ in the radiation-dominated era to zero in the matterdominated era, changing appreciably only since the era of radiation-matter equality, $1+z_{e q}=1 / a_{e q}=3880$. (We are assuming a flat Universe with $h^{2}=0.5$ [9], so that at present, the critical density $\rho_{B}+\rho_{Q}$ is $0.940 E-29 \mathrm{~g} \mathrm{~cm}^{-3}=4.05 E$ $-47 \mathrm{GeV}^{4}$, the quintessence-matter ratio is $\mathcal{R}_{0}=2$, and the background density is $\rho_{B 0}=1.35 E-47 \mathrm{GeV}^{4}$.)

In the next three sections, we consider tracking scalar field dynamics generally, the parametrization of two different dynamical quintessence models, and other possibilities for the smooth energy. In Sec. V, we review a recent calculation [10] showing that, for $\mathcal{R}_{0} \sim 2$, the asymptotic collapsed mass fraction is maximal so that the evolution of intelligent observers is most likely. Finally, this observation is used to infer anthropically and heuristically a testable constraint on 
future theories of quantum cosmology and the static or quasistatic nature of the smooth energy.

\section{SCALAR FIELD DYNAMICS: DISTINGUISHING DYNAMIC FROM STATIC SMOOTH ENERGY}

The equation of motion for the scalar field follows from the flat-space Friedmann equation

$$
H^{2}=(8 \pi G / 3)\left(\rho_{B}+\rho_{Q}\right),
$$

and two coupled equations for quintessence energy conservation and for the evolution of $\rho_{Q}(a)$ and $\phi(a)$

$$
\begin{aligned}
-d \rho_{Q} / d N & =3 \dot{\phi}^{2}=6\left(\rho_{Q}-V\right)=n_{Q} \rho_{Q} \\
\frac{d \phi}{d N} & =(\dot{\phi} / H)=\sqrt{6\left(\rho_{Q}-V\right) /(8 \pi G \rho)} \\
& =\sqrt{n_{Q} \Omega_{Q}} \cdot M_{P} .
\end{aligned}
$$

On the right-hand side, $\rho=\rho_{Q}+\rho_{B}, \rho_{Q}=\dot{\phi}^{2} / 2+V$, and $P$ $=\dot{\phi}^{2} / 2-V$. The two dependent variables $\rho_{Q}$ and $\phi$ are functions of the independent variable $N \equiv \ln a$. Hereafter, we use reduced Planck units $8 \pi G \equiv M_{P}^{-2}=1$ for $\phi$.

Using $V(\phi)=\rho_{Q}\left(1-w_{Q}\right) / 2$ and defining [8] a function

$$
\begin{aligned}
\Delta(a) & \equiv d \ln V / d \ln \rho_{Q}=1+d \ln \left(1-w_{Q}\right) / d \ln \rho_{Q} \\
& =1+\left(d w_{Q} / d N\right) / 3\left(1-w_{Q}^{2}\right),
\end{aligned}
$$

we can rewrite Eq. (3) as

$$
\begin{aligned}
d w_{Q} / d N & =3\left(1-w_{Q}^{2}\right)(\Delta-1), \quad \text { where } \\
\Delta & =\left(-V^{\prime} / V\right) \sqrt{\Omega_{Q} / n_{Q}} .
\end{aligned}
$$

Defining $x \equiv \dot{\phi}^{2} / 2 V(\phi)$, the ratio of quintessence kinetic and potential energies, Eq. (6) shows $\Delta(a)-1=d \ln x /$ $6 \cdot d N$ to be appreciable only where the quintessence field is changing rapidly. Thus $w_{Q}$ varies slowly where the field is either nearly frozen $\left(w_{Q} \sim-1\right)$ or is tracking $(\Delta \sim 1)$.

Starting from a broad range of initial conditions, $w_{Q}(\phi)$ oscillates between $\sim 1$ and $\sim-1$ and enters an epoch in which the energy density $\rho_{Q}(\phi)$ is frozen at a relatively small value. Then, this field converges onto a tracker solution in which the slope $-d \ln \rho_{Q} / d N \equiv n_{Q}$ is nearly constant at a value less than $-d \ln \rho_{B} / d N \equiv n_{B}$ for the background matter. Here $N \equiv \log a$, and $n_{i} \equiv 3\left(1+w_{i}\right)=-d \ln \rho_{i} / d N$ is the adiabatic index: $n_{B}=4$ and 3 for radiation and matter, respectively.

\section{PARAMETRIZATION OF DYNAMICAL QUINTESSENCE BY TWO DIFFERENT EQUATIONS OF STATE}

The condition for a tracker solution to exist is [8] that $-V^{\prime} / V$ be a slowly decreasing function of $\phi$ or $N$, where $V^{\prime} \equiv d V / d \phi$, or that

$$
0<\Gamma(a)-1 \equiv d\left(-V / V^{\prime}\right) / d \phi
$$

be nearly constant. It is convenient to measure the steepness of the potential by the logarithmic derivative $\beta(\phi)$ $\equiv-d \ln V / d \ln \phi$, so that

$$
\Gamma-1=d(\phi / \beta) / d \phi=1 / \beta(\phi) \cdot(1-d \ln \beta / d \ln \phi) .
$$

We will consider below, an inverse-power potential for which $\beta$ is strictly constant, and an "isothermal" potential, for which $\beta$ slowly decreases with $\phi$ so as to keep the equation of state $w_{Q}=$ const.

The ordinary inflationary parameters are then

$$
\eta(\phi) \equiv \frac{V^{\prime \prime}}{V}, \quad 2 \epsilon \equiv\left(\frac{V^{\prime}}{V}\right)^{2}, \quad \Gamma=\frac{V \ddot{V}}{\dot{V}^{2}}=\frac{\eta(\phi)}{2 \epsilon(\phi)} .
$$

When $\eta, 2 \epsilon \ll 1$, a tracking potential will be slow-rolling, meaning that $\ddot{\phi}$ in the scalar field equation of motion and $\dot{\phi}^{2}$ in the kinetic energy are both negligible. This slow-roll approximation is usually satisfied in ordinary inflation. In the early e-folds of quintessence, however, $-V^{\prime} / V=\beta(\phi) / \phi$ is slowly changing but is itself not small for $\beta \neq 0$. This establishes the important distinction between static or quasi-static quintessence $\left[n_{Q}(1) \sim 0, w_{Q 0} \sim-1\right]$ and sensibly dynamical quintessence $\left(n_{Q}>1, w_{Q}>-2 / 3\right)$. At the observationally allowed upper limit $w_{Q 0}=-0.5$, the first few scale e-folds are fast-rolling. Such dynamical quintessence will become slowroll only in the very far future, after many e-folds of the quintessence field slowly driving $\Delta$ from 1 to zero and $w_{Q}$ from its present value to zero. This means that while the slow-roll approximation is applicable to ordinary inflation, dynamical quintessence generally requires exact solution of the equations of motion (4) and (6).

For tracking potentials derived from physical principles, it is reasonable to assume that $\beta \approx$ const, at least since tracking began. We therefore parametrize quintessence so that the SUSY-inspired potential

$$
V(\phi)=V_{0}\left(\phi_{0} / \phi\right)^{\beta}, \quad \beta=3.5
$$

is inverse power law, and $\Gamma-1=1 / \beta=$ const.

For comparison, we also consider the isothermal equation of state

$$
V(\phi)=V_{0}\left[\sinh \left(\alpha \phi_{0}\right) / \sinh (\alpha \phi)\right]^{\beta},
$$

where $\beta=2, \alpha \equiv \sqrt{3 /\left(2 \beta+\beta^{2}\right)}=0.612$. Then $\Gamma-1=\Omega_{B} / \beta$ is not constant, but decreases from $1 / \beta$ when tracking begins, to zero in the far future. This potential is called "isothermal" because for it, $w_{Q}=-2 /(2+\beta)=-1 / 2$ and $n_{Q}=3 \beta /(2$ $+\beta)=3 / 2$ are constant once matter dominates over radiation. This makes the tracker approximation exact for the isothermal potential: $\Delta=1$, Eq. (6) is trivially satisfied, and Eq. (4) makes $-V^{\prime} / V=n_{Q} \cdot d a / a$. This potential allows a scaling solution $V(a), \rho_{Q}(a) \sim a^{-n_{Q}}$ [11] and has the inverse power potential $V=V_{0}\left(\phi_{0} / \phi\right)^{\beta}$ and the exponential potential $V$ $=V_{0} \exp \left(-\sqrt{n_{Q}} \phi\right)$ as limits for $\alpha \phi \ll 1$ and $\gg 1$, respectively. It therefore interpolates between an inverse power potential at early times and an exponential potential at late times. 


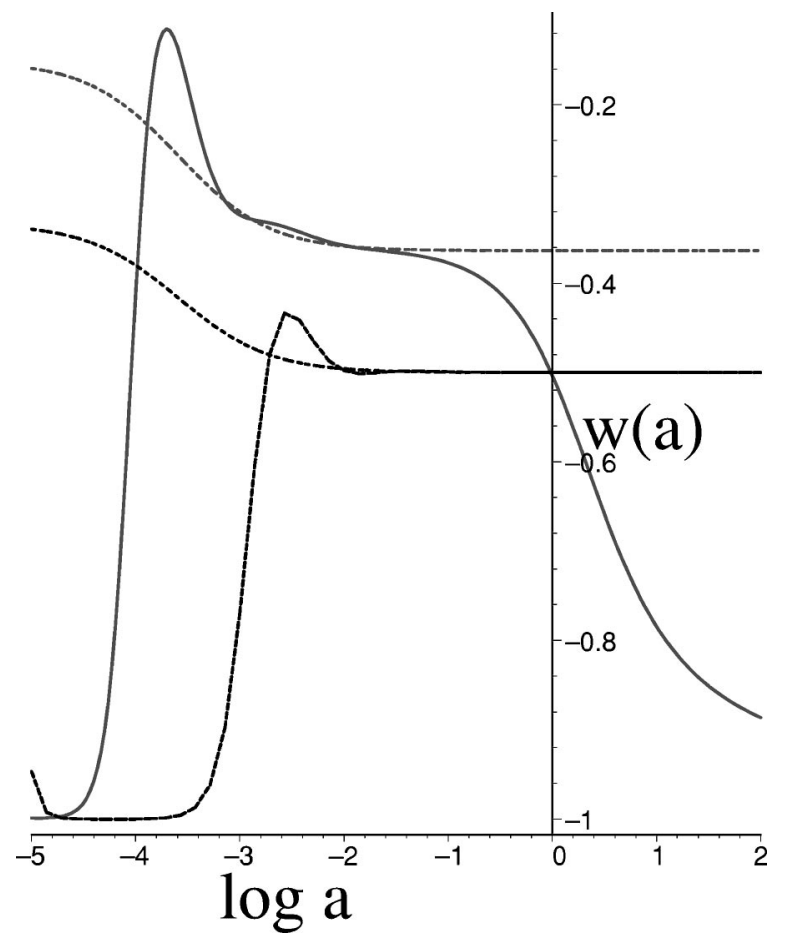

FIG. 1. Exact and tracker approximation solutions $w_{Q}$ for inverse power and isothermal potentials chosen to give present values $\mathcal{R}_{0}=2, w_{Q 0}=-0.5$. The exact $w_{Q}$ values for the inverse-power and the isothermal quintessence potentials (10) and (11) are shown by the solid and dashed curves. The tracker approximation (dashdotted curves) is exact for the isothermal potential, but holds only briefly for the inverse-power potential and considerably overestimates $w_{Q}$ once quintessence is appreciable.

In each of these potentials, the two parameters have been chosen to fit the present values $\mathcal{R}_{0}=2$ and $\rho_{Q 0}=2 \rho_{c r} / 3$ $=2.7 E-47 \mathrm{GeV}^{4}$, so that for $w_{Q 0}=-0.5, n_{Q 0}=3 / 2$ the present value of the potential is $V_{0}=V\left(\phi_{0}\right)=1.013 E$ $-47 \mathrm{GeV}^{4}$. This requires tracking to begin after matter dominance, reaching present values $\phi_{0}=2.47$ for the inverse-power and 1.87 for the isothermal EOS, respectively. Tracking continues indefinitely for the isothermal EOS, but is of relatively short duration for the inverse-power potential (Fig. 1). We believe these parametrizations of $V(\phi)$ to be representative of reasonable smooth potentials, over the redshift range $z<5$ that is observationally accessible. If we considered lower values of $\beta$ for the present ratio $\mathcal{R}_{0}=2, w_{Q 0}$ would decrease from $-1 / 2$ to -1 as the potential became faster rolling.

For the inverse-power potential, $-V^{\prime} / V=\beta / \phi$, and for the isothermal potential $-V^{\prime} / V=\beta \alpha / \tanh (\alpha \phi)$, respectively, so that, for $w_{Q 0}=-0.5, \eta$ and perforce $\epsilon$ are not now small. Thus both the dynamical quintessence potentials we are considering are now still fast-rolling. The isothermal potential, with $\beta=2$, asymptotically approaches $\exp (-1.22 \phi)$ and will never be slow-rolling. The inverse-power potential, with $\beta=3.5$, will become slow-rolling when $\phi>\beta$ and will asymptotically approach a de Sitter solution in the distant future. In the observable recent past, its $w_{Q}$ increases with $z$ approximately as $w_{Q}(z) \approx-0.5+0.016 z$.

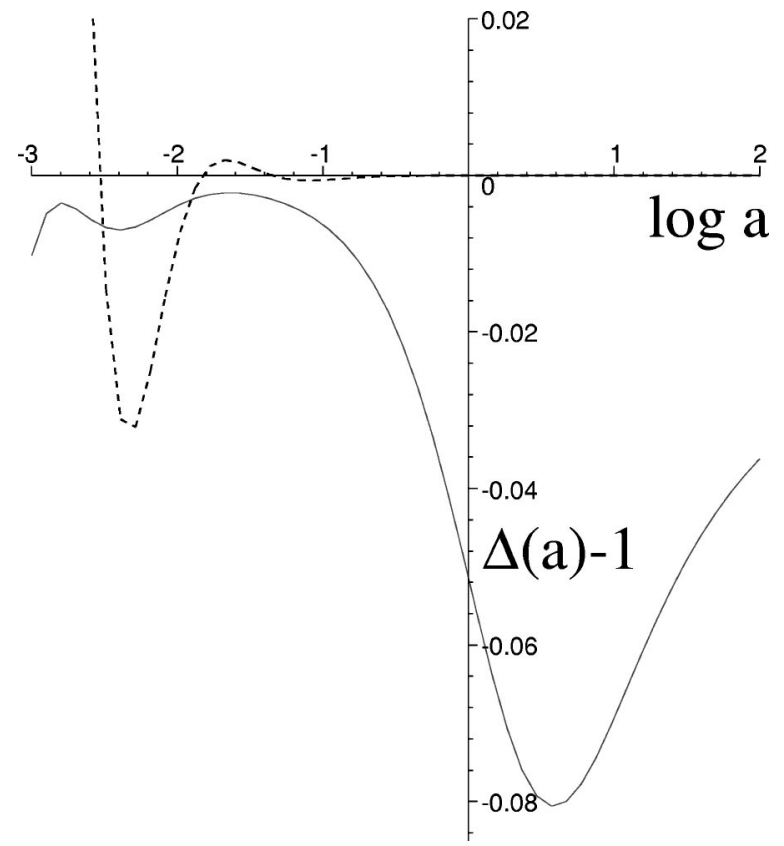

FIG. 2. The quantity $\Delta-1=d \ln x / 6 \cdot d \ln a$ for the inversepower and the isothermal quintessence potentials (10) and (11) are shown by the solid and dashed curves. $\Delta$ differs from unity only before tracking and, in the case of the inverse-power potential, where the quintessence field is growing.

\section{A. Tracker approximation}

After transients depending on initial conditions, and a frozen epoch $\left(w_{Q} \approx-1\right)$, the scalar field overshoots and then converges onto a solution

$$
n_{Q}(a)=n_{B}(a) \beta /(2+\beta)=n_{B}(a) / 2
$$

which tracks the background $n_{B}(a)$ (Fig. 1). During the tracking regime, until quintessence dominates, $\Delta \approx 1$ and $\Omega_{Q} \approx n_{Q} \phi^{2} / \beta(\phi)$ increases quadratically with field strength.

Driven by the background EOS which is changing around $z_{c r}=3880$, the quintessence equation of state parameter $w_{Q}(a)$ slowly decreases. The inverse-power potential reaches its tracker at $\log a \approx-3.2$, but remains there only until $\log a \approx-1.8$, when the growth of the quintessence field slowly drives $w_{Q}$ down from the tracker value -0.364 towards -1 in the very far future (solid curve in Fig. 1). For the inverse-power potential, the tracker approximation holds only briefly, and thereafter seriously overestimates $w_{Q}$. The exact isothermal solution reaches its tracker later, at $\log a$ $>-1$, but remains exactly on tracker thereafter (dashed curve in Fig. 1).

Figure 2 magnifies the changes in $w_{Q}$ that transpire before and after tracking, by plotting $d \ln x / 6 \cdot d N$, where $x$ is the ratio of quintessence kinetic to potential energy. Figure 3 shows the integrated effects of $n_{Q}(a)$, by plotting the integral of Eq. (3).

Once reaching the tracker, the isothermal equation of state stays constant at $w_{Q}(\phi)=-2 /(2+\beta)=-1 / 2$. The evolution with cosmological scale is fixed by the scaling $V / V_{0}$ $=\rho_{Q} / \rho_{Q 0}=a^{-3 / 2}$, which makes (cf. Fig. 4) 


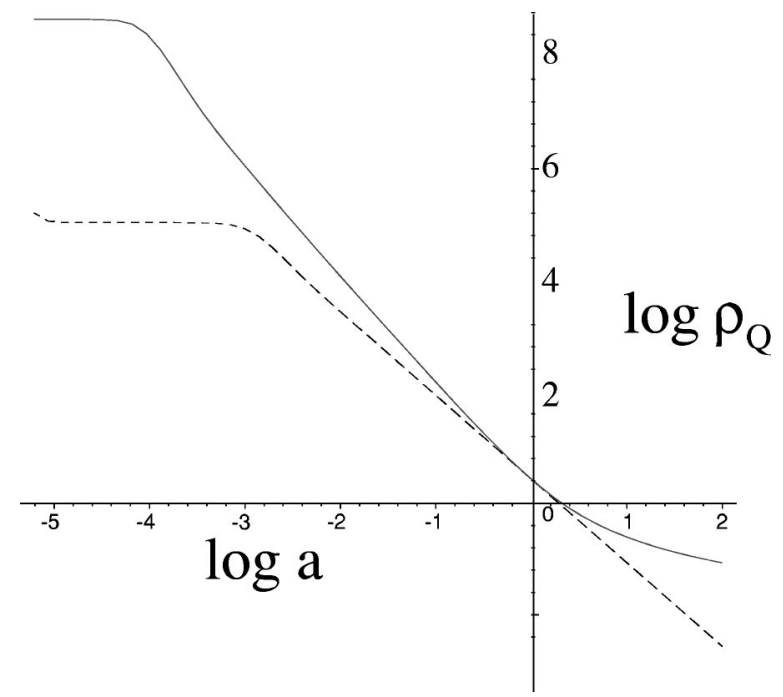

FIG. 3. Logarithmic plots of the quintessence energy density, in units $\mathrm{GeV}^{4}$, for the inverse-power (solid curve) and isothermal (dashed curve) potentials. After transients, but before tracking, the fields are frozen. On tracker, the adiabatic index in $\rho_{Q} \sim a^{-n_{Q}(a)}$ stays at $3 / 2$ for the isothermal EOS, but continuously decreases towards zero for the inverse-power EOS. The present density $\rho_{Q 0}$ $=2 \rho_{c r 0} / 3$ and adiabatic index $n_{Q 0}=1.5$ are the same for both.

$$
\mathcal{R}(a)=\sinh ^{2}(\alpha \phi), \quad \Omega_{Q}=1-\Omega_{B}=\tanh ^{2}(\alpha \phi) .
$$

For the present ratio $\mathcal{R}_{0} \sim 2$ one has $\alpha \phi_{0} \leqslant 1.146$ and $\phi_{0} \leqslant 1.87$. The observations that the Universe is now accelerating [6] fix the bounds

$$
-2 /(2+\beta) \leqslant-1 / 2, \quad \beta \leqslant 2, \quad \alpha \geqslant 0.612, \quad \phi_{0} \leqslant 1.87 .
$$

For $\beta=0$, the potential is static: $\Lambda$-dominance started at redshift $R_{0}^{1 / 3}-1=0.260$, the universe was then already accelerating $-q=0.333$, and is now accelerating faster $-q_{0}=0.5$. For larger $\beta$, the scalar field is more dynamic. At the upper limit, $\beta=2$ the isothermal potential rolls only as fast as the observed acceleration $\left(-q_{0} \geqslant 0\right)$ allows, tracking starts only after matter domination, and acceleration starts only now. Nevertheless, $\rho_{Q}(a)$ was still subdominant to the radiation density all the way back into the era of big bang nucleosynthesis [12]. In Sec. V, we will consider these two limits for the isothermal smooth energy $\beta=2$ (QCDM) and $\beta=0$ (LCDM).

As mentioned above, the tracking approximation is exact for the isothermal potential, but only briefly valid for the inverse-power potential. For $\alpha \phi \ll 1$, the background dominates and the isothermal equation of state reduces to the inverse power potential $V \sim \phi^{-\beta}$, for which, along the tracker, $\phi, \sqrt{\mathcal{R}} \sim a^{3 /(2+\beta)}$. We shall, however, also be interested in the quintessence dominated era $\alpha \phi>1$, when different potentials give different present and future behavior.

\section{B. Present and future of the quintessence-dominated universe}

According to Eq. (6), the quintessence field is frozen at $w_{Q} \approx-1$ just before tracking, and, except for the isothermal potential, also in the very far future.

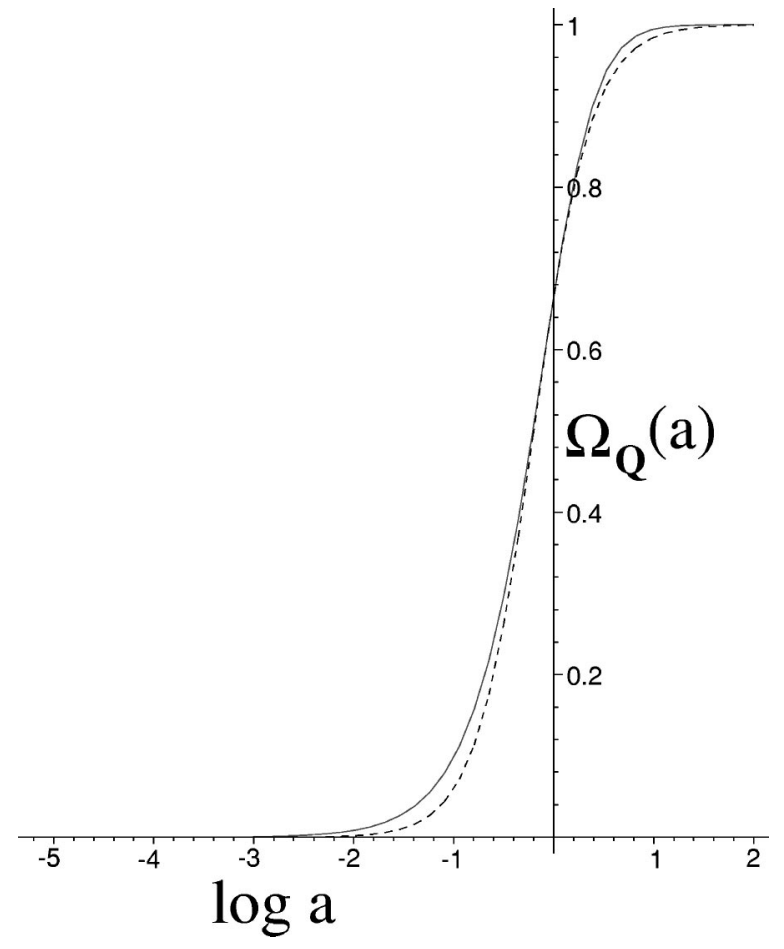

FIG. 4. Evolution of the quintessence fraction of the energy density. $\Omega_{Q}(a)$ starts growing as $n_{Q} \phi^{2} / \beta(\phi)$ at tracking, reaches $2 / 3$ and is changing most rapidly at present. It will reach unity only in the distant future.

The difference between the two potentials (10),(11) appears only in the evolution of $w_{Q}$ now and in the future, when $\alpha \phi>1$.

For the inverse power potential (and generically), the growth of quintessence drives $\Delta(a)$ below unity and $w_{Q}(a)$ decreases slowly towards -1 . The quintessence energy density $\rho_{Q}$ and the expansion rate $H \rightarrow$ const, drive ultimately towards a de Sitter universe, which inflates exponentially, with no event horizon. The asymptotic behavior of our isothermal EOS potential is atypical: it will approach the exponential potential, $w_{Q}$ stays constant, $\rho_{Q}$ and $H$ continue to decrease, and inflation is power law. Atypically, the isothermal EOS will always exhibit an event horizon and may not derive from string theory.

Figure 4 shows the evolution of the quintessence fraction of the total energy density $\Omega_{Q}(a)$ from 0 to 1 , presently passing through $\Omega_{Q 0}=2 / 3$. This evolution is similar for both potentials, although the inverse-power potential is more dynamic than the isothermal EOS.

\section{OTHER POSSIBILITIES FOR SMOOTH ENERGY}

The inverse-power potential (10) is a reasonable phenomenological description for potentials which are not decreasing too fast at present. The isothermal potential $w_{Q}=$ const is as good as any other potential we might choose phenomenologically in the near future, and it allows an analytic solution.

If $w_{Q}$ is not constant, we could still reconstruct the quintessence potential from $w_{Q}(z)$. Indeed $d w_{Q}(z) / d z$ is generically positive for quintessence and generally negative for 
$k$-essence [13], an alternative in which the scalar field has a non-linear kinetic energy instead of a potential. In principle, $w_{Q}(z)$ is observable in high-redshift supernovae [1], in cluster evolution [14,15] and in gravitational lensing [16]. In practice, $w_{Q}(l)$ is poorly constrained observationally [5] and theoretically [17-19]. Over the small redshift range for which smooth energy dominates and for which sensitive measurements are possible, the effects of varying $w_{Q}(z)$ are much smaller than the present uncertainties in measurement and in cosmological model. Theoretically, the luminosity distance to be measured in high-redshift supernovae and in comoving volume number density measurements depends on integrals which smooth out the sensitivity to $w_{Q}(z)$ [18]. Until $\Omega_{m 0}$ is determined to (3-2)\% accuracy, SNAP [20] and other future experiments will only be able to determine some $w_{Q}$ effective over the observable redshift range $z<2$, to tell whether the smooth energy is static or dynamic. If we wait long enough, the differences among different quintessence potentials will asymptotically become substantial.

In the next section, we will study how different structure evolves to the present $\mathcal{R}_{0}=2$ for $w_{Q}=-1$ (LCDM) and for $w_{Q}=-1 / 2(\mathrm{QCDM})$. We should interpret this constant $w_{Q}$ $=-1 / 2$ chosen as some maximal average value of $w(z)$. The present maximal value $w_{Q 0}$ would then be somewhat less than $-1 / 2$ for quintessence and somewhat greater for $k$-essence.

\section{EVOLUTION OF LARGE SCALE STRUCTURE}

Since structure evolves only in a matter-dominated universe, its evolution started about the time of radiation-matter equality and ended about matter-quintessence equality $\mathcal{R}$ $=1$ or at scale $a=\mathcal{R}_{0}^{1 / 3 w} Q$ i.e. at redshift $\mathcal{R}_{0}^{1 / 3}-1$ $=0.260, \mathcal{R}_{0}^{2 / 3}-1=0.53$ for LCDM and for our isothermal $w_{Q}=-1 / 2$ QCDM respectively.

Using an improved Press-Schecter formalism [10], holding fixed all other dimensionless constants, we calculated as function of $\mathcal{R}_{0}$ the fraction of mass that would have already collapsed into structures larger than 1-2 Mpc. We interpret this asymptotic collapsed mass fraction as a measure of the relative likelihood of large-scale structures, galaxies, habitable solar systems and intelligent life. (There is a tacit assumption here that intelligent life depends critically on the evolution of large scale structure and is therefore relatively rare in our universe.) Not surprisingly [21], the observed $\mathcal{R}_{0} \sim 2$ fell within the range of small cosmological constants or smooth energy density for which intelligent life is likely. Surprisingly, this likelihood was maximal for our QCDM and nearly maximal for LCDM.

\section{THE ANTHROPIC AS A HEURISTIC PRINCIPLE}

Quintessence explains how the cosmological "constant" $\Omega_{Q}$ could become small, but not why it is just now comparable to $\Omega_{B}$ and the Universe is now accelerating. Hopefully, the one remaining cosmological parameter $\mathcal{R}_{0} \sim 2$ will someday be derived from fundamental theory. Meanwhile, in the face of what is now known, what is the least biased inference to be made from the observed datum $w_{Q} \sim 2$, at or near the peak of the logarithmic asymptotic mass distribution?

Applying probability considerations to a unique universe is problematic. Nevertheless, we have now become accustomed to the role of observers in quantum mechanics. This encourages us, at least tentatively, towards an anthropic explanation of the cosmic coincidence, why we live at a time when the smooth and clustered energy densities are approximately equal. Following Vilenkin's principle of mediocrity [22], we assume that, out of a conceivable ensemble of flat cosmologies, our universe or cosmology is typical of those permitting intelligent life. Then the observed datum suggests that the entire prior distribution in $\mathcal{R}_{0}$ must be flat, at least for $\mathcal{R}_{0}=\mathcal{O}(1)$. But, if we are decided to apply probability to the Universe, over what is the prior a distribution?

Proponents of the anthropic principle $[21,22]$ have assumed a prior distribution of real universes, with different values of $\mathcal{R}_{0}$, almost all of which are inhospitable to life. Since these many other universes are practically unobservable, this application has no practicable predictive value, and is rightfully criticized as unscientific. It is important, however, to remember that the prior is a functional of (subjective) hypotheses, not of the data sample.

Instead of such a prior distribution of universes, we [10] assumed a flat prior probability distribution,

$$
\mathcal{P}_{*} \propto 1 /\left(-V^{\prime}(\phi)\right) \quad \text { slowly varying, }
$$

for (future) fundamental theories. This does not require that the potential be slow-rolling ( $\eta, \epsilon \ll 1)$. If prospective measurements show $w_{Q 0} \approx-1$, then Eq. (15) requires a quintessence potential of generic form like $V(\phi)=V_{1} f(\lambda \phi)$, where $V_{1} \sim \mathcal{O}\left(M_{P}^{4}\right)$ is a large energy density, $f(x)$ is a dimensionless function involving no very large or very small parameters, and $\lambda$ is a very small dimensional parameter [23]. Because such a prediction for the static or quasistatic quintessence potential is theoretically and observationally falsifiable, our way of applying anthropic reasoning to theories is scientific, while the application to subuniverses is not, at least not until these other universes can be observed. This application of anthropic reasoning is only heuristic, as was the equivalence principle which guided us towards general relativity and the correspondence principle which guided us towards quantum mechanics.
[1] A.G. Riess et al., Astron. J. 116, 1009 (1998); S. Perlmutter et al., ibid. 517, 565 (1999).

[2] M.S. Turner and A.G. Riess, astro-ph/0106051.

[3] P. de Bernardis et al., astro-ph/0105296; R. Stompor et al., astro-ph/0105062; C. Pryke et al., astro-ph/0104490.

[4] S.M. Harun-or-Rashid and M. Roos, Astron. Astrophys. 373,
369 (2001).

[5] P. Garnavich et al., Astrophys. J. 509, 74 (1998).

[6] N. Bahcall, J. Ostriker, S. Perlmutter, and P. Steinhardt, Science 284, 1481 (1999); A. Balbi et al., Astrophys. J. Lett. 547, L89 (2001).

[7] A.D. Dolgov, in The Very Early Universe, edited by G.W. Gib- 
bons, S.W. Hawking, and S.T.C. Siklos (Cambridge University Press, Cambridge, England, 1983), p. 449; P.J.E. Peebles and B. Ratra, Astrophys. J. Lett. 325, L17 (1988); B. Ratra and P.J.E. Peebles, Phys. Rev. D 37, 3406 (1988); C. Wetterich, Nucl. Phys. B302, 668 (1988).

[8] P.J. Steinhardt, L. Wang, and I. Zlatev, Phys. Rev. D 59, 123504 (1999).

[9] W.L. Freedman et al., Astrophys. J. 553, 47 (2001).

[10] S. Bludman and M. Roos, Astrophys. J. 547, 77 (2001).

[11] L.A. Ureña-López and T. Matos, Phys. Rev. D 62, 081302 (2000); T. Matos and L.A. Ureña-López, ibid. 63, 063506 (2001).

[12] R. Bean, S.H. Hansen, and A. Melchiorri, Phys. Rev. D 64, 103508 (2001).

[13] C. Armendariz-Picon, V. Mukhanov, and P.J. Steinhardt, Phys. Rev. Lett. 85, 4438 (2000).

[14] J.A. Newman and M. Davis, Astrophys. J. Lett. 534, L11
(2000).

[15] G.P. Holder et al., Astrophys. J. 544, 629 (2000); Z. Haiman, J.J. Mohr, and G.P. Holder, ibid. 553, 545 (2001).

[16] A.R. Cooray and D. Huterer, Astrophys. J. Lett. 513, L95 (1999); A.R. Cooray, Astron. Astrophys. 342, 353 (1999).

[17] V. Barger and D. Marfata, Phys. Lett. B 498, 67 (2001).

[18] I. Maor, R. Brustein, and P.J. Steinhardt, Phys. Rev. Lett. 86, 6 (2001).

[19] P. Astier, astro-ph/0008306.

[20] J. Weller and A. Albrecht, astro-ph/0106079.

[21] S. Weinberg, Phys. Rev. Lett. 59, 2607 (1987).

[22] G. Efstathiou, Mon. Not. R. Astron. Soc. 274, L73 (1995); M. Tegmark and M.J. Rees, Astrophys. J. 499, 526 (1998); J. Garriga, M. Livio, and A. Vilenkin, Phys. Rev. D 61, 023503 (2000).

[23] S. Weinberg, Phys. Rev. D 61, 103505 (2000). 